\title{
THE SPECTRUM OF A FUNCTION ALGEBRA
}

\section{ROBERT GEORGE BLUMENTHAL ${ }^{1}$}

The spectrum of a function algebra $A$ defined on a space $X$ is sometimes obtained by attaching a "thin" set to $X$. Function algebras with this property have been constructed for various purposes by J. Wermer [5], S. J. Sidney [4], and E. Kallin [2]. We present here a theorem which reveals the underlying basis for this phenomenon. Theorem 1.1 shows that to compute the spectrum of a function algebra $A$ on $X$ it is sometimes sufficient to compute the spectrum of $\mathrm{CL}(A \mid F)$, where $F$ is the set of common zeros in $X$ of an ideal of $A$, and then attach it to $X$ along $F$.

A function algebra $A$ is a closed point-separating subalgebra of $C(X)$ containing the constants, where $X$ is a compact Hausdorff space. The spectrum of $A$, denoted by $S(A)$, is the compact Hausdorff space of nonzero complex homomorphisms $\phi$ of $A$ taken in the Gelfand topology. We regard $X$ as a closed subspace of $S(A)$. $A$ extends via the Gelfand transform to a function algebra on $S(A)$, and, when convenient, we shall regard $A$ as a function algebra on $S(A)$.

1. Let $A$ be a function algebra and $F$ a closed subset of $S(A)$. We set for $f \in A,\|f\|_{F}=\sup \{|f(\phi)|: \phi \in F\}$. The $A$-convex hull of $F$, denoted $A$-hull $(F)$, is by definition

$$
\left\{\phi \in S(A):|f(\phi)| \leqq\|f\|_{F}, \text { all } f \in A\right\} .
$$

$F$ is said to be $A$-convex if $F=A$-hull $(F) . A$-hull $(F)$ can be identified with the spectrum of the restriction algebra $\operatorname{CL}(A \mid F)$. If $I$ is an ideal of $A$, then $\{\phi \in S(A): f(\phi)=0$, all $f \in I\}$ is called the hull of $I$ with respect to $A$, and is denoted by hull ${ }_{S(A)} I$, or simply hull- $I$. hull $_{S(A)} I$ is nonempty if, and only if, $I$ is proper, and is obviously $A$-convex.

TheOREm 1.1. Let $A$ and $B$ be function algebras on $X=S(A)$. Suppose $I \subseteq A \cap B$ is a closed ideal of both $A$ and $B$, and let $F=$ hull $_{S(A)} I$. Then $S(B)=S(A) \cup_{F} S(\mathrm{CL}(B \mid F))$, i.e. the space obtained by attaching $S(\mathrm{CL}(B \mid F))$ to $S(A)$ along $F \subseteq S(A)$.

Before proving Theorem 1.1 we recall two well-known facts about ideals.

Received by the editors May 23, 1969 and, in revised form, November 3, 1969.

${ }_{1}$ The material of this paper is taken from the author's doctoral thesis (Yale University, 1968) written under the direction of Professor Charles E. Rickart. 
Lemma 1.2. Let $A$ be a function algebra, and let $I$ be a closed ideal in $A$. Then:

(a) Every nontrivial homomorphism of $I$ is given by evaluation at a point of $S(A)$-hull- $I$.

(b) If $\phi$ and $\bar{\phi}$ are in $S(A)$ and either $\phi$ or $\bar{\phi}$ is not in hull-I, then there exists a function $f$ in I such that $f(\phi) \neq f(\bar{\phi})$.

Proof. For (a), see [3, Theorem (3.1.18)]; (b) is trivial.

Proof of Theorem 1.1. We have to prove

(i) $B$-hull $(F) \cap S(A)=F$, and

(ii) if $\phi \in S(B)-S(A)$ then $\phi \in B$-hull $(F)-S(A)$.

Since (i) is clear, it suffices to prove:

(1.1) If $\phi \in S(B)-S(A)$, then $|f(\phi)| \leqq\|f\|_{F}$ for every $f \in B$. We show first

(1.2) $S(B)-S(A) \subseteq$ hull $_{S(B)} I$.

Let $\phi \in S(B)-S(A)$ and suppose $\phi \notin$ hull $_{S(B)} I$. Then $\phi$ induces a nontrivial homomorphism of $I$ by point evaluation. Now since $I$ is an ideal of $A$, Lemma 1.2(a) implies the existence of an element $\bar{\phi} \in S(A)-F$ such that $f(\phi)=f(\bar{\phi})$ for all $f \in I$. I, however, is also an ideal in $B$ and since $\phi \notin$ hull $_{S(B)} I$, Lemma 1.2(b) implies that $\bar{\phi}=\phi$ $\in S(B)-S(A)$, which contradicts the fact that $\bar{\phi} \in S(A)$.

To prove (1.1) note that $S(B)-S(A)$ is an open subset in the complement of the Šilov boundary for $B$. (1.2) implies that the topological boundary of $S(B)-S(A)$ lies in $S(A) \cap$ hull $_{S(B)} I=F$. (1.1) now follows by an application of Rossi's local maximum modulus principle $[1$, p. 63].

There is a simple way of constructing of pair of function algebras $A, B$ which satisfy the hypothesis of Theorem 1.1. If $A_{0}$ and $A$ are function algebras on $X=S(A), A_{0} \subseteq A$, and $I$ is an ideal of $A$, we may take $B=\mathrm{CL}\left(A_{0}+I\right)$. Sidney's theorem is concerned with algebras constructed in this fashion, although the algebras $A_{0}$ and $A$ in this instance are tensor products of other function algebras.

If $A$ and $B$ are subalgebras of $C(X)$ and $C(Y)$ respectively, by $A \otimes B$ we mean the closure in $C(X \times Y)$ of the algebra generated by functions of the form $f \otimes g, f \in A, g \in B$, where $f \otimes g(x, y)=f(x) g(y)$. When $A$ and $B$ are function algebras, $A \otimes B$ is a function algebra on $X \times Y$ and $S(A \otimes B)=S(A) \times S(B)$.

Theorem 1.3 (S. J. Sidney [4]). Let $A$ and $A^{\prime}$ be function algebras on $X=S\left(A^{\prime}\right)$ where $A \subseteq A^{\prime}$, let $B$ be a function algebra on $Y=S(B)$, and let $I$ be a closed ideal of $B$. Then $\mathfrak{U}=\mathrm{CL}\left(A \otimes B+A^{\prime} \otimes I\right)$ is a function algebra on $X \times Y$, and $S(\mathfrak{H})=(X \times Y) \cup(S(A) \times$ hull- $I)$. 
Proof. $A \otimes B$ is a subalgebra of $A^{\prime} \otimes B$ and $A^{\prime} \otimes I$ is an ideal of $A^{\prime} \otimes B$. By the preceding discussion $\mathfrak{A}$ is a subalgebra of $A^{\prime} \otimes B$ which contains an ideal $A^{\prime} \otimes I$ of $A^{\prime} \otimes B$. If we set $F=$ hull $_{X \times Y} A^{\prime} \otimes I$, then $F=X \times$ hull- $I . \quad$ By Theorem 1.1, $S(\mathfrak{A})=(X \times Y) \cup_{F} S(\mathrm{CL}(\mathfrak{A} \mid F))$. Since $\mathfrak{A}|F=A \otimes B| X \times$ hull- $I, S(\mathrm{CL}(\mathfrak{A} \mid F))=S(A) \times$ hull- $I$, completing the proof.

Wermer's example is stated in terms of polynomial convexity. The polynomial convex hull of a compact subset $K$ of $\boldsymbol{C}^{n}$, denoted by $p$-hull $(K)$, is by definition

$$
\left\{z \in \boldsymbol{C}^{n}:|f(z)| \leqq\|f\|_{K} \text {, all polynomials } f\right\} .
$$

If $A$ is a finitely generated function algebra on $X$ with generators $h_{1}, \cdots, h_{n}$, then the mapping $H: S(A) \rightarrow C^{n}$ defined by setting $H(\phi)=\left(h_{1}(\phi), \cdots, h_{n}(\phi)\right)$ is a homeomorphism of $S(A)$ into $C^{n}$ such that $H(S(A))=p$-hull $(H(X))$.

Now let $X=\left\{\left(z_{1}, z_{2}\right) \in C^{2}:\left|z_{i}\right| \leqq 1, i=1,2\right\}$, let $A$ be the uniform closure of the polynomials on $X$, so that $S(A)=X$, and let $B$ be the subalgebra of $A$ (containing the constants) generated by $z_{1}, z_{1} z_{2}$, $z_{1} z_{2}^{2}-z_{2}$. Then $B$ is a function algebra on $X$ which contains the principal ideal $I$ of $A$ generated by $z_{1} z_{2}-1$ [5, Lemma 4]. Define $\Phi: X \rightarrow C^{3}$ by $\Phi\left(z_{1}, z_{2}\right)=\left(z_{1}, z_{1} z_{2}, z_{1} z_{2}^{2}-z_{2}\right)$. Setting $F=h l_{1} I$, we see that $F=\{(z, \bar{z}):|z|=1\}$, so that $\Phi(F)=\{(z, 1,0):|z|=1\}$. Theorem 1.1 and the above remarks now imply the main result of $[5] ; p$-hull $(\Phi(X))$ $=\Phi(X) \cup\{(z, 1,0):|z|<1\}$.

2. If $A$ is a function algebra, a function $f \in C(S(A))$ is said to be locally in $A$ if there exists an open covering $\left\{U_{\lambda}: \lambda \in \Lambda\right\}$ of $S(A)$ such that $f\left|U_{\lambda} \in A\right| U_{\lambda}$, all $\lambda \in \Lambda$. $A$ is called a nonlocal function algebra if there exists a function which is locally in $A$ but not in $A$. The question of the existence of nonlocal function algebras was settled by Eva Kallin who constructed an example of such an algebra [2].

This example can be conveniently presented in terms of Sidney's construction. Let $D=\{z \in C:|z| \leqq 1\}, R=\{z \in C: 1 \leqq|z| \leqq 2\}, T_{i}$ $=\{z \in C:|z|=i\}, i=1,2$ and for $K$ equal to $D$ or $R$ let $A(K)$ denote the algebra of all continuous functions on $K$ which are analytic in the interior of $K$. Let $I$ be the principal ideal of $A(D)$ generated by $z^{2}$, and let $A_{R}=A(R) \mid T_{1} \cup T_{2}$. Then Kallin's example can be identified with the subalgebra of $C\left(\left(T_{1} \cup T_{2}\right) \times D\right)$ given by

$$
\mathrm{CL}\left(A_{R} \times A(D)+C\left(T_{1} \cup T_{2}\right) \times I\right) .
$$

This identification leads to the construction of a whole class of 
nonlocal function algebras; one can show, by generalizing the proof of the nonlocalness of this algebra, that when certain simple restrictions are imposed on the constituent algebras of Sidney's theorem, the resulting algebra is nonlocal.

\section{BIBLIOGRAPHY}

1. R. C. Gunning and H. Rossi, Analytic functions of several complex variables, Prentice-Hall, Englewood Cliffs, N. J., 1965. MR 31 \#4927.

2. E. Kallin, A nonlocal function algebra, Proc. Nat. Acad. Sci. U.S.A. 49 (1963), 821-824. MR $27 \# 2878$.

3. C. E. Rickart, General theory of Banach algebras, The University Series in Higher Math., Van Nostrand, Princeton, N. J., 1960. MR 22 \#5903.

4. S. J. Sidney, Properties of the sequence of closed powers of a maximal ideal in a sup-norin algebra, Trans. Amer. Math. Soc. 131 (1968), 128-148. MR 36 \#5701.

5. J. Wermer, An example concerning polynomial convexity, Math. Ann. 139 (1959), 147-150. MR 22 \#12238.

University of Miami, Coral Gables, Florida 33124 